\title{
La littérature francophone ou l'art de s'approprier la langue de l'Autre
}

\author{
Judith Sinanga-Ohlmann \\ University of Windsor
}

\begin{abstract}
Résumé
Le présent article tente de montrer comment les auteurs francophones d'Afrique sub-saharienne s'approprient la langue française, idiome appartenant à l'Autre puisque hérité de l'excolonisateur. Cette pensée d'en être propriétaire ou de se l'approprier se heurte évidemment contre celle de Jacques Derrida qui a brillamment défendu l'idée qu'une langue ne saurait être une chose que l'on peut posséder et sur laquelle il serait possible d'avoir un contrôle quel qu'il soit. Notre article n'a pas l'objectif de contredire l'argument de Jacques Derrida, a contrario. En effet, si une langue ne peut être un objet auquel on peut prétendre avoir un droit quelconque, elle est néanmoins une matière malléable et les locuteurs non natifs peuvent, soit l'adopter comme ils l'ont apprise ou la transformer selon leur culture, leurs croyances, leur philosophie, etc. Lui faire subir des changements aussi bien du point de vue lexicologique, morphologique, syntaxique, etc., afin de l'adapter à leurs us et coutumes est ce que nous entendons par appropriation de la langue de l'Autre. Pour quelles raisons les auteurs francophones d'Afrique sub-saharienne entreprennent-ils de s'approprier le français ? Parviennent-ils à faire leur cette langue ? Par quels moyens ? Telles sont quelques-unes des questions posées dans cet article et auxquelles nous avons essayé de répondre en nous fondant sur trois romans: Le chercheur d'Afriques (Henri Lopes, 1990), Quand on refuse on dit non (Ahmadou Kourouma, 2004) et La femme aux pieds nus (Scholastique Mukasonga, 2008).
\end{abstract}

Mots-clés : Langues, littérature, cultures, dominants, dominés 
Le présent article vise à montrer la manière dont les romanciers francophones d'Afrique sub-saharienne parviennent, à travers leurs œuvres de fiction, à faire de la langue française un outil malléable qu'ils transforment volontairement afin de l'acclimater aux habitudes langagières et aux us et coutumes des $«$ lecteurs africains ${ }^{26}$. Ce passage du français standard au français à résonnances africaines ou «français africain », ainsi que l'a soutenu Pierre Dumont (1990: 8-9) ${ }^{27}$, est ce que nous qualifions d'acte d'appropriation de la langue de l'Autre. Par quels moyens et par quelles techniques discursives les auteurs francophones d'Afrique sub-saharienne parviennent-ils à réussir cet exploit ? Pour répondre à cette question, il a été nécessaire de faire une étude comparative entre la grammaire du français standard et celle qui a été privilégiée par lesdits auteurs dans les passages de leurs textes où ils ont senti le besoin d'exprimer leurs pensées dans un ton et accent typiquement africains. À cet effet, trois œuvres ont été retenues : Le chercheur d'Afriques (Henri Lopes, 1990), Quand on refuse on dit non (Ahmadou Kourouma, 2004) et La femme aux pieds nus (Scholastique Mukasonga, 2008).

Le chercheur d'Afriques a été plus largement utilisé dans notre étude, ceci dû au fait que son auteur y a déployé des efforts considérables pour que le langage de ses personnages de fiction ne trahisse aucunement la manière dont s'expriment les peuples réels que ces derniers sont censés représenter. Force est de reconnaître qu'il a brillamment mené à bien ce projet en réinventant la langue française aussi

\footnotetext{
${ }^{26}$ Nous parlons ici de « lecteurs africains » à cause de la réponse que nous a donnée Henri Lopes quand, lors d'un entretien qu'il nous a accordé, Chantal Grosso et moi-même lui avons demandé ce que vise un auteur en introduisant des mots et accents africains dans son livre. Il a répliqué : «Il essaye de faire un roman africain. Il ne peut pas écrire dans la langue africaine qu'il parle. Il cherche à ce qu'un Africain qui ouvre son livre ne soit pas perdu ».

${ }^{27}$ Nous tenons cette information de Danièle Latin (1995 : 163) qui a cité Pierre Dumont dans son article: "Particularités lexicales et variétés du français: l'enjeu de l'instrumentalisation lexicographique. À propos des inventaires du Sud et du français en Afrique Noire ».
} 
bien du point de vue lexicologique, morphologique que syntaxique. C'est dans l'ensemble sur ces trois points que nous nous sommes attardée et non sur la technique d'oralité que Ladislas Nzesse (2010 : 247) a défini comme une parole qui s'énonce sous forme de maximes, de chants, de contes ou de proverbes. Quoique nous reconnaissions le fait que ces derniers soient une des manières dont l'auteur africain s'approprie la langue française, notre article vise plutôt à montrer de quelle façon les textes du corpus matérialisent un parler français typiquement africain ainsi que Pierre Dumont l'a décrit en disant :

Le français d'Afrique n'est pas une invention de linguistes en mal d'imagination. C'est une réalité avec laquelle il faut maintenant compter. Il existe un français régional africain aux nombreuses variétés [...] Langue de l'innovation référentielle, le français est en train de devenir le véhicule de valeurs expressives spécifiquement africaines, le lieu de production d'un sens africain, le berceau d'un véritable et nouvel univers sémiotique. (op. cit. : 1)

Ce que Pierre Dumont désigne comme « un français régional africain aux nombreuses variétés » nous semble aller au-delà du phénomène d'oralité décrite précédemment et il ne s'aligne pas non plus exactement avec la simple qualification de «français populaire » que Ladislas Nzesse a également désigné comme une des manifestations d'oralité dans l'écriture (Ibid.: 248). Nous pensons que l'acte de s'approprier la langue de l'Autre n'en serait pas véritablement un si on le limitait sur ce concept d'oralité. C'est, nous semble-til, dans la reconnaissance que la langue de l'Autre, en passant des locuteurs d'une région à une autre, se diversifie ou devient une variante que l'idée d'appropriation se concrétise. Le chercheur d'Afriques a donc été spécifiquement choisi pour montrer qu'il existe des variétés, ou « variationnisme » (Jean-Nicolas de Surmont, 2008 : 361) dans la langue française. 
Pour ce qui est du roman Quand on refuse on dit non, nous nous en sommes intéressée suite à la manière dont Ahmadou Kourouma a inondé ce texte de définitions sémantiques, de traductions en français des mots en langue vernaculaire et des explications de proverbes et dictons africains. Quel était le but de Kourouma derrière cette méthode narratologique ? En quoi cette technique scripturale peut-elle être mise en relation avec l'acte d'appropriation de la langue de l'Autre ? Telles sont les deux pistes que nous explorons dans le deuxième pan de cet article.

Quant au roman La femme aux pieds nus, il a été inclus au corpus à cause du refus de son auteur d'employer des mots de la langue française qui ne traduisent pas exactement le sens ainsi que les connotations culturelles de certains vocables du kinyarwanda, sa langue maternelle. De ce rejet des mots français a résulté le procédé de translation qui consiste à faire passer un mot d'une langue donnée dans une autre. La technique de translation comme manière de faire sienne la langue française, mais aussi comme façon de ne pas pervertir les mots intraduisibles de la langue maternelle de l'auteur, est ce dont nous discutons en dernier lieu.

Cependant, avant d'entrer dans le vif du sujet, peut-on être propriétaire d'une langue ? D'après l'argument de Jacques Derrida fondé sur l'expérience de la communauté juive d'Algérie dont il faisait partie et qui s'est vu du jour au lendemain retirer la nationalité française, une langue ne saurait être la propriété d'un État ni d'une personne. Ainsi en a-t-il témoigné dans son œuvre Le monolinguisme de l'Autre ou la prothèse d'origine: 
[...] le maître ne possède pas en propre, naturellement, ce qu'il appelle pourtant sa langue ; parce que, quoi qu'il veuille ou fasse, il ne peut entretenir avec elle des rapports de propriété ou d'identité naturels, nationaux, congénitaux, ontologiques ; parce qu'il ne peut accréditer et dire cette appropriation qu'au cours d'un procès non naturel de constructions politico-phantasmatiques; parce que la langue n'est pas son bien naturel, par cela même il peut historiquement, à travers le viol d'une usurpation culturelle, c'est-àdire toujours d'essence coloniale, feindre de se l'approprier pour l'imposer comme « la sienne ». (Jacques Derrida, $1996: 45$ )

Comment pouvons-nous alors soutenir que les auteurs francophones font du français leur propre langue si même celui qui les a colonisés et dont ils l'ont héritée ne pourrait, selon les propos de Derrida, prétendre en être le possesseur?

Dans le présent article, la suggestion que l'auteur francophone s'approprie la langue de l'Autre n'implique évidemment pas le sens de posséder en propre mais correspond plutôt à cette définition de K. Ploog et B. Rui, citée par Ladislas Nzesse (2010 : 256) : «s'approprier signifie, s'agissant d'un code linguistique, faire sien. Faire la langue sienne, c'est agir à travers elle ; c'est toujours, à travers elle, construire son identité individuelle et sociale». Faire sien l'idiome de l'Autre réfère donc au procédé de l'adapter/l'acclimater aux habitudes langagières des pays et peuples mis en scène dans l'œuvre littéraire. Telle est la démarche que Lopes admet adopter dans ses œuvres ainsi qu'il en a témoigné dans un entretien accordé à Édouard Maunick :

Au départ, je pose et je dis que je vais parler congolais, que je vais parler congolais en français, en écrivant dans cette langue d'emprunt que j'aime. [...] Ma grande préoccupation n'est pas de raconter une histoire qui serait un procès-verbal de police ou un documentaire journalistique mais de me raconter avec le piment que j'ai dans mes entrailles. (Henri Lopes, 1988 : 129) 
Lopes a-t-il réussi à rester fidèle à cette devise qu'il s'est fixée ? Qu'est-ce qui a amené cet écrivain qui a fait sa formation scolaire en France et qui a été élevé par un homme français ${ }^{28}$ à décider d'écrire ses œuvres de fiction dans un français d'Afrique que ceux qui se croient comme propriétaires légitimes ${ }^{29}$ décrivent en termes de «barbarismes»? Il est important ici de rappeler la réponse que l'auteur congolais a donnée quand on lui a demandé pourquoi il écrivait. Il a dit :

En écrivant Tribaliques, je pensais m'adresser d'abord aux Africains, notamment à mes compatriotes, les Congolais. La réception que vous lui avez réservée m'a fait prendre conscience qu'une voix africaine, exprimant une société et des cultures éloignées, pouvait éveiller des échos sur un autre continent. Sans doute en l'écrivant, caressais-je ce désir sans en avoir une claire conscience, $\mathrm{j}$ 'en avais une intuition confuse. (Allocution prononcée à l'occasion de l'obtention d'un doctorat honoris causa, le 26 avril 2002, Université Laval)

Si en composant Tribaliques il pensait s'adresser à ses compatriotes et n'était pas certain de son désir d'éveiller des échos sur un autre continent, dixneuf ans plus tard, son écriture dans Le Chercheur d'Afriques témoigne d'un écrivain sûr de lui-même et de son but de faire transparaître à travers son œuvre ses origines, son « moi » authentique. Ce roman dont le thème central est pourtant

${ }^{28}$ Nous tenons ces informations de l'entretien que Céline Gahungu (2018) a eu avec Henri Lopes. Celui-ci lui a confié son expérience des années passées dans un internat à Nantes, ville dont il parle beaucoup dans son roman Le chercheur d'Afriques.

${ }^{29}$ L'idée nous vient de l'article de Laude Ngadi Maïssa (2019 : 199) où on témoigne : « Il n’y a plus de notre langue française. Notre langue est en berne. Comme nous ne croyons plus en elle, le reste du monde non plus » (NLF, 171). Or, ajoute Delacomptée, l'adjectif possessif « notre » marque le lien affectif du peuple français à sa langue et exprime «le sentiment d'une appartenance » (24) patriotique et identitaire qui impose à l'écrivain « la garde » (196) d'une réalité qui «possède une identité, un esprit, un "génie", qui réclame qu'on la respecte sous peine de la violer ». 
la quête d'identité est en même temps la confirmation de cette dernière grâce à l'écriture et au langage adoptés par son auteur. En effet, à travers le discours direct dans les nombreux dialogues du roman, la communication entre différents personnages a servi de prétexte à Lopes pour faire une reproduction fidèle du français local.

\section{S'exprimer avec le piment de ses entrailles}

Pour répondre à la question au sujet de la devise lopésienne de parler congolais en français, considérons quelques passages du roman Le chercheur d'Afriques :

1) Elle m'a bahattu !

Dans les bras de ma mère je montrai la coupable du doigt.

- L'enfant-là !

Olouomo se lança dans un débit rapide, à en perdre le souffle, à dire tout le mal que j'avais fait ; que... ; que...; et puis que... ;

Elle termina par un bruit de bouche insultant.

- Tu vois, tu vois, elle m'a tchipé.

- L'enfant-là, vraiment ! (18)

2) - Veux dire, hein, que quand tu bois l'eau de la dame-jeanne-là tu deviens sorcier tellement ta tête elle est devenue savante.

- Or que c'est comme ça ?

- Comme ça, même ! de l'eau grigri !

- Quand tu as bu l'eau de Mpoto-là, tu deviens pour toi gaillard, gaillard, plus gaillard que les guerriers du Ngalien*30.

- Ahan?

- Tu peux gifler sans problème le nègre le plus fier.

\footnotetext{
${ }^{30}$ Les mots qu'il a fait suivre d'astérisque ont dû, apparemment à la demande de l'éditeur, être expliqués en notes infrapaginales. Nous tenons de la remarque suivante l'idée que c'est l'éditeur qui l'a exigé : «Une fois encore, mon éditeur, décidément trop européocentriste, veut me forcer à expliquer. Selon lui, le lecteur français ne peut comprendre qu'il s'agit du féminin de voyou ». (Henri Lopes, 1990 : 216).
} 
Si tu le tapes, il te regarde comme un homme ligoté par le diable, et la fièvre le saisit. Il dit merci seulement. S'il est capable de répondre, le tonnerre gronde et le dissout automatiquement en des milliers de morceaux plus petit que le sel fin. Immédiatement, là même, sur place, comme je te dis !

[...] - Alors tu le bottes élégamment... On vient, on le ramasse, on le jette et on pleure matanga* jusqu'à quand la nuit fatiguée demande pardon et s'en va pour elle.

- Ahan?

- Vrai de vrai!

- Yéhé !

Et nous posions nos mains sur nos bouches.

La palabre s'était interrompue. Ngantsiala et ma mère se retirèrent, abandonnant là le Commandant, son interprète, le boy éventail, le boy dame-jeanne et les mboulou-mboulous. (85-86)

3) - Ne cherche pas à comprendre, l'homme. Laisse seulement, je vais te raconter après.

La dernière phrase avait été dite en lingala. [...] (168)

Nous avons dans ces citations des exemples qui traduisent une manière de s'exprimer qui est typique dans certains pays d'Afrique francophone subsaharienne. On constate dans la première citation l'ajout de l'interjection « là » à la fin d'un mot ou d'une phrase tel que dans ces énoncés : «L'enfant-là !» et « L'enfant-là, vraiment ! ». Dans ce contexte, la particule « là » ne renvoie pas à l'idée de ce qui est éloigné mais exprime plutôt la désapprobation d'Olouomo envers Andélé ; l'enfant qui se méconduit. Il en est de même de l'adverbe « vraiment » qui termine le second morceau et dont le sens ici ne réfère pas à ce qui est vrai mais plutôt à ce qui est incroyable. C'est aussi de cette façon qu'en certains cas on manifeste son mécontentement ou on communique un reproche à quelqu'un. Un autre fait à signaler est le néologisme «tchiper» qui, comme le narrateur l'explique, est un « bruit de bouche insultant». Tchiper est un mot qui décrit un geste en même temps que le bruit qui l'accompagne. C'est sans doute à 
cette manière de s'exprimer que réfère Ramuz qui a confié à Lise Gauvin (2010 : 9) que ce qu'il « cherche avant tout c'est l'expressivité de la parole, sa couleur, ou mieux encore, ce qu'il appelle une «langue-geste », capable de rendre les accents et les rythmes de son pays, de les convertir en équivalents littéraires ».

En dehors de ce néologisme, les passages cités ci-dessus contiennent également des phrases dont la syntaxe ne respecte pas celle du français standard. Observons-en, entre autres, un exemple dans la réplique suivante : «- Ne cherche pas à comprendre, l'homme. Laisse seulement, je vais te raconter après ». Le mot «l'homme » mis en apposition dans la phrase précédente paraît ambigu pour un locuteur non congolais. Sans la virgule qui sépare ce mot du reste de la phrase, cette remarque aurait complètement un sens différent. Pour empêcher un lecteur non averti de penser que cette phrase est ambiguë ou agrammaticale, l'auteur, sans néanmoins en expliquer le sens, a ajouté cette clarification : «La dernière phrase avait été dite en lingala »(Henri Lopes, 1990 : 168). Nous nous trouvons ici en face d'une traduction littérale d'une phrase dite en lingala et que Lopes a choisi de transférer dans son texte en français sans changer la structure de la langue d'origine. Cet acte délibéré de faire une traduction littérale d'une phrase dite en lingala constitue un autre exemple d'appropriation du français puisque sa structure syntaxique a été transformée pour accommoder celle du lingala.

Mis à part le choix de transformer la langue française en construisant des phrases dont la syntaxe reproduit plutôt celle de l'un ou l'autre des idiomes congolais, l'auteur s'est efforcé de donner à ces dialogues un rythme qui reflète un ton et un accent particuliers tel que dans le passage suivant: «Alors tu le bottes élégamment... On vient, on le ramasse, on le jette et on pleure matanga* 
jusqu'à quand la nuit fatiguée demande pardon et s'en va pour elle. - Ahan ? Vrai de vrai ! - Yéhé !» (Henri Lopes, 1990 : 86).

Non seulement on devine un accent particulier à travers la manière dont la phrase a été scandée, mais aussi Lopes y a introduit des interjections telles que «Ahan», "Yéhé» qui sont de l'oralité africaine. Le ton fabulateur qui caractérise ce passage rappelle aussi une manière typiquement africaine de raconter un récit. Au ton oral et à l'accent local, il a joint des mots en langues congolaises comme le matanga, les mboulou-mboulous ou encore des termes congolais francisés comme «le Mouroupéen» et d'autres fois des mots composés dont le sens se réfère à des faits socio-culturels, voire historiques, particuliers. On peut, à titre d'exemple, citer : «le boy éventail» et «le boy dame-jeanne » (Henri Lopes, $1990: 85)$.

Le boy éventail fait référence à celui qui, pendant la période coloniale, devait se tenir à côté d'un maître colonisateur pour assumer la fonction de rafraichir l'air et de chasser les insectes loin de ce dernier en utilisant un grand éventail. Quant au boy dame-jeanne, c'était celui qui portait une dame-jeanne contenant de quoi boire pour le colonisateur. Nous avons donc ici des mots qui non seulement rappellent une certaine époque, mais aussi un statut social de quelqu'un.

L'on pourrait considérer les vocables tels que les mboulou-mboulous ${ }^{31}$, les mikatés (Henri Lopes, 1990 : 35), le matanga (Ibid.: 86), etc., comme des néologismes dans la mesure où les traduire en langue française reviendrait à fausser leur sens car ils connotent certaines réalités culturelles qu'une simple

\footnotetext{
${ }^{31}$ Mot qui au Rwanda signifie fil barbelé, au Congo-Brazzaville, il réfère aux tirailleurs.
} 
traduction ne parviendrait pas à rendre dans la langue de l'Autre. À titre d'exemple, le mot matanga que l'auteur a traduit en bas de page en termes de veillée funèbre subit une réduction de sens car veillée funèbre ne renvoie pas nécessairement à l'acte collectif de pleurer. On a, dans certains pays d'Afrique, lors de ces veillées, des femmes qu'on appelle des pleureuses. Celles-ci ne font pas que verser des larmes, elles le font en poussant des cris stridents d'où l'expression «pleurer matanga » employée dans le roman. Si donc un Français devait parler d'une telle veillée dans le contexte congolais, pour en rendre toutes les connotations, il lui faudrait dire comme Lopes «pleurer matanga». Sans doute est-ce en partie à de telles particularités langagières que Calixthe Beyala faisait allusion quand elle a dit que «le français est francophone mais la francophonie n'est pas française ${ }^{32}$. Par-dessus le rejet de la domination française par ses ex-colonies, on peut aussi déduire de sa remarque que la France ne saurait avoir aucun contrôle sur la langue qu'elle leur a léguée ${ }^{33}$. Ainsi, introduire et approuver dans le lexique du français de tels termes serait accepter le fait que la langue de Molière est, par voie de circonstances, plurielle et, qu'en tant que telle, elle doit déborder les frontières de l'Hexagone.

Le chercheur d'Afriques est certes un roman qui ne respecte pas souvent la grammaire du français standard, mais nous croyons qu'il est juste de dire que ses lecteurs, qu'ils soient d'origine africaine ou d'ailleurs, n'en sont pas pour autant perdus. C'est dire qu'en s'appropriant la langue de l'Autre, Lopes ne l'a pas transformée en un charabia (Lilyan Kesteloot, 1999 : 45) incompréhensible mais

\footnotetext{
${ }^{32}$ Exergue du roman Les honneurs perdus (1996).

${ }^{33}$ Ainsi l'a-t-elle confirmé en disant : «...si le tronc commun de la langue française se trouve en France, il y a bien fort longtemps que des rejetons de l'arbre français ont poussé partout dans le monde, que la langue s'est diversifiée, tout en demeurant une, qu'elle s'est batomanioquée au son des tam-tam, qu'elle s'est modifiée au son des balafons et même de la rumba Congolaise... » (Calixthe Beyala, Dîner-Débat au cercle Richelieu Senghor de Paris, 4 février 2020).
} 
l'a enrichie par des tournures qui reflètent une identité spécifique, une pensée et une façon d'agir propres à un peuple particulier. Cela est bien dépeint dans les phrases suivantes : « Olouomo frappa de son index une joue invisible. » (Lopes, 1990 : 18), «Et celui qui nous tenait sous le charme de son savoir se tranchait la gorge de l'index, qu'il secouait pour en faire tomber la goutte de sang » (Ibid. : 86).

Ce procédé de joindre le geste à la parole est ce qu'André-Patient Bokiba a désigné comme «affaire de langage ». Selon lui, «au-delà de la langue, la littérature est aussi affaire de langage ; elle inclut un arsenal de signifiants qui ne sont pas tous verbaux » (propos cités par Raymond Mopoho, 2017). Qu'en est-il de l'utilisation de la langue de l'Autre chez Kourouma?

\section{Quand on refuse on dit non ou signe de «l'altérité littéraire »}

Selon Raymond Mopoho (2017), l'altérité littéraire dans le domaine de la traduction est l'acte délibéré de la part d'un écrivain de faire ce qu'il veut de la langue de l'Autre. Il nous a semblé approprié d'appliquer ce concept au roman Quand on refuse on dit non du fait de nombreuses instances où cette fiction cesse d'être un récit qu'on narre pour devenir une sorte de classe où les apprenants sont amenés à observer un enseignant qui se plaît à manipuler le sens des mots qu'il leur apprend et à s'adonner à un jeu dans lequel le français et les langues africaines se chevauchent. Par ce moyen, l'auteur a prouvé qu'il est possible pour les auteurs d'Afrique noire francophone d'écrire en français sans perdre ni leur lectorat d'Afrique ni celui de France ou d'autres pays francophones du monde. Il en a témoigné, de façon ironique certes, en faisant dire à Birahima, son narrateur enfant-soldat, les propos suivants : 
[...] j'ai quatre dictionnaires pour me débarbouiller et expliquer les gros mots qui sortent de ma petite bouche. Larousse et Petit Robert pour le français français des vrais Français de France ; le Harrap's pour le pidgin (le pigdin est une langue composite née du contact commercial entre l'anglais et les langues indigènes) ; l'inventaire des particularités lexicales du français d'Afrique noire pour les barbarismes d'animistes avec lesquels les nègres d'Afrique noire de la forêt et de la savane commencent à salir, à noircir la limpide et logique langue de Molière. Le Larousse et le Petit Robert permettent d'expliquer le vrai français français aux noirs animistes d'Afrique noire. L'inventaire des particularités lexicales du français en Afrique noire essaie d'expliquer aux vrais français français les barbarismes animistes des noirs d'Afrique. (Ahmadou Kourouma, 2003 :19)

Viser et atteindre un public diversifié - les Français de France, les francophones d'Afrique et ceux d'ailleurs, telle paraît être la devise de Kourouma dans la citation ci-dessus. Néanmoins, on ne peut pas ne pas remarquer la manière ironique dont il l'annonce surtout dans le passage où il parle de l'acte de salir et de noircir la limpide et logique langue de Molière. Nous retrouvons dans ce dernier roman de Kourouma un écrivain qui n'a pas oublié le fait qu'un prix littéraire ait été refusé à son œuvre Les soleils des indépendances à cause de sa façon, lui a-t-on dit, de traiter la langue française. C'est peut-être à la suite de cette expérience que la décision d'adopter une technique scripturale définitoire lui est venue. Mais comment satisfaire tous les membres de la francophonie sans trahir les habitudes langagières et culturelles des uns ou des autres? Peut-être que la solution, pour l'auteur africain, qu'il décide d'écrire en français malinké ou en français congolais, etc., l'essentiel est que son œuvre n'en soit pas indécodable. Il nous semble que c'est bien à cela que fait référence la remarque suivante : 
La seule limite que connaît le travail d'aliénation que l'écrivain africain effectue sur sa langue d'emprunt demeure celle que lui impose la compréhension de son texte. Pour Henri Lopes, la littérature : 'autorise à aller jusqu'où l'on veut dans l'usage de la langue dans la mesure où la compréhension est assurée. La seule limite imposée à l'écrivain tient donc à la compréhension : dans cette limite, il est libre de bousculer les codifications et de tordre la langue. (Attisoh et Adama, 2003 : 21)

\section{« La seule limite imposée à l'écrivain tient à la compréhension »}

Cette remarque de Kouévi Attisoh est fort à propos en ce qui concerne les deux derniers romans de Kourouma, Allah n'est pas obligé et Quand on refuse on dit non. En effet, contrairement à ses autres textes où les lois de la langue française ont été délibérément transgressées sans que l'auteur fournisse des explications quelles qu'elles soient, le procédé de définir ou expliquer des mots français compliqués mais aussi de traduire des termes malinkés, etc., semble avoir été une autre nouveauté ${ }^{34}$ dans la production littéraire de Kourouma. Son roman Quand on refuse on dit non en est un bon exemple. Plusieurs pages de cette œuvre contiennent une grande quantité de mots qui sont définis de façon classique, c'est-à-dire comme on s'y attendrait dans un dictionnaire, alors que d'autres, en revanche, servent de prétexte à l'auteur pour exprimer un fait culturel particulier ou émettre un jugement politique. Observons, par exemple, la manière dont il a expliqué l'expression «enfant de développé » (Ahmadou Kourouma, 2004 :14). Comme il n'est pas dit dans le roman que l'explication vient d'un dictionnaire ou de l'inventaire des particularités africaines, nous en avons déduit que «enfant de développé » est peut-être sa propre création. Cet assemblage de

\footnotetext{
${ }^{34}$ Une autre nouveauté car, comme l'a dit Lilyan Kesteloot (1999: 42), Kourouma a été le premier écrivain francophone à transgresser les codes linguistiques de la langue du colonisateur. Il l'a affirmé lui-même lors de son entretien avec Yves Chemla lorsqu'il a dit qu'avec Allah n'est pas obligé, il a introduit une forme nouvelle dans la littérature négro-africaine.
} 
mots signifie, d'après Birahima : « ressortissant d'un pays développé. Un pays du Nord où il fait froid, où il y a de la neige » (Ibid. : 14). Il est difficile de ne pas voir en cette définition une comparaison qui rappelle le statut économique des pays du Nord - développés -, et ceux du Sud - en voie de développement - d'où l'idée de jugement politique justifié par le désir de Birahima de devenir un enfant de développé. Avoir ajouté « où il fait froid, où il y a de la neige » n'est pas non plus un acte gratuit. Un(e) autre mot/expression qu'il a expliqué(e) est « enfant de la préférée » (Ibid. : 33). L'auteur aurait pu dire que Birahima est traité comme un « enfant préféré » mais il a choisi d'utiliser une expression qui n’éloigne pas son récit des habitudes culturelles de la communauté mise en scène dans le roman Quand on refuse on dit non où il est question de la coutume polygamique. Même si nous n'avons cité que ces deux exemples, le roman en offre beaucoup d'autres dont les définitions /explications peuvent être considérées comme des paratextes car elles vont plus loin que ce que ces vocables signifient pour faire corps avec le récit. Cette technique nous semble corroborer l'idée d'altérité littéraire en ce sens que la langue de l'Autre est utilisée de façon inventive ou déformée pour satisfaire au besoin de l'auteur de communiquer un fait particulier. Qu'en est-il dans le roman La femme aux pieds nus de Scholastique Mukasonga?

\section{Quand parler de soi dans la langue de l'Autre devient impossible}

Ce roman qui parle d'une communauté de Tutsi du Rwanda qui, après la chute de la monarchie tutsie en 1959, s'est retrouvée expropriée de ses biens puis déportée dans une région du pays où il était difficile de vivre, met l'emphase sur des habitudes culturelles qui étaient propres à ces gens. Dans le roman, l'insertion des mots de la langue maternelle semble corroborer ce fait et a donc comme fonction la valorisation culturelle des exilés. On peut exiler quelqu'un, on peut 
lui arracher sa nationalité mais on ne peut pas lui prendre la langue ; tel est le témoignage de Jacques Derrida dans son Monolinguisme de l'Autre ou la prothèse d'origine (1996). Quand tout bien matériel a été pris, la langue est ce qui reste, la langue est le moyen par lequel on dira sa nostalgie du lieu perdu.

La femme aux pieds nus, comme les deux autres romans du corpus, comprend beaucoup de mots en langue maternelle que l'auteur a traduits en français pour en expliquer le sens au lecteur. Cependant, certains termes de la langue maternelle de l'auteur ont été transférés dans la langue française sans subir des transformations pour empêcher que leur portée culturelle n'en soit pervertie. On le voit dans le passage où Mukasonga évoque le mot «amata » qui signifie lait en français. Mais le terme français « lait» pour le peuple rwandais et plus particulièrement les personnes de l'ethnie tutsi historiquement connues comme peuple pasteur ne saurait contenir les mêmes connotations que le mot utilisé dans la langue maternelle. Il nous semble que c'est pour cette raison que l'auteur a tenu à le dire aussi en kinyarwanda. Il en a été de même pour le mot « inzu »dont elle a catégoriquement rejeté la traduction en français par les vocables « hutte, cahute, paillotte ». En témoignent ces propos :

C'est peu de temps après notre installation à Gitagata que Stefania décida que le temps était venu de construire, derrière la case de Tripolo, l'inzu, la maison qui était pour elle aussi nécessaire que l'eau pour le poisson ou l'oxygène pour les humains. Non qu'elle acceptât désormais sa condition d'exilé - jamais elle ne s'y résigna - mais elle savait que c'était de cette demeure originelle qu'elle puiserait toujours la force nécessaire pour faire face au malheur et renouveler l'énergie qu'elle dépensait [...] La maison de Stefania, celle où elle pouvait vivre une vraie vie de femme, une vraie vie de mère de famille, c'était la maison de paille tressée comme une vannerie, l'inzu (et je lui garderai son nom en kinyarwanda, car je n'ai rien en français 
que des noms de mépris pour la désigner : hutte, cahute, paillotte...).

(Scholastique Mukasonga, 2008 : 40)

Le refus d'utiliser un autre mot que l'inzu, la désinvolture que l'on ressent dans l'explication de ce refus est ce qui nous a fait dire qu'il arrive quelquefois des moments où parler de soi ou de sa culture dans la langue de l'Autre devient impossible, d'où le recours au procédé de translation. Peut-on parler dans ce cas d'appropriation de la langue de l'Autre ? Oui, sans doute, dans la mesure où on montre que cette dernière a des limites. Ainsi, l'appropriation du français par les auteurs africains sert aussi, dans certaines circonstances, à empêcher la perversion des langues vernaculaires. Sinon comment les dominés pourraient-ils empêcher le danger d'annihilation de certains éléments culturels mais aussi des codes langagiers intraduisibles s'ils les exprimaient toujours selon la logique des langues de ceux qui les ont colonisés ? 


\section{Bibliographie}

Beyala, Calixthe, Dîner-Débat au cercle Richelieu Senghor de Paris, 4 février 2020. https://www.cercle-richelieu-senghor.org/2020/02/04/mme-calixthebeyala-le-francais-et-les-francais/ [le 21 juillet 2021].

Beyala, Calixthe, Les honneurs perdus, Paris, Albin Michel, 1996.

Chemla, Yves, «Entretien avec Ahmadou Kourouma », Notre Librairie, Paris, CLEF, 1998, p. 135. http://ychemla.net/fic_doc/kourouentret.html [le 5 juillet, 2017].

Constant, Isabelle, "Quand on refuse on dit non: Roman du dire cruel, ou Comment écrire la guerre?», Nouvelles Études Francophones, vol 22, $\mathrm{n}^{\circ} 2$, Automne 2007, pp. 34-43.

Coulibaly, Adama, «Allah n'est pas obligé ou la Parole injurieuse », Nouvelles Études Francophones, vol 22, n² ${ }^{\circ}$, Automne 2007, pp. 11-24.

Derrida, Jacques, Le Monolinguisme de l'Autre ou la prothèse d'origine, Paris, Éditions Galilée, 1996.

Gahungu, Céline, « Sculpter la matière, écrire le monde : entretien avec Henri

Gauvin, Lise, «Entre rupture et affirmation: les manifestes francophones », Études littéraires africaines, $\mathrm{n}^{\mathrm{o}} 29,2010$, pp 7-14.

Kesteloot, Lilyan, «Négritude et créolité », dans Christiane Albert (dir.), Francophonie et identités culturelles, Paris, Karthala, 1999, pp. 39-48.

Kouévi Attissoh, Clément Adama, Aspects du roman francophone négroafricain post-indépendance, Lille, ANRT, 2003.

Kourouma, Ahmadou, Allah n'est pas obligé, Paris, Éditions du Seuil, 2000.

Kourouma, Ahmadou, Les soleils des indépendances, Paris, Éditions du Seuil, 1970.

Kourouma, Ahmadou, Quand on refuse on dit non, Paris, Éditions du Seuil, 2004.

Latin, Danièle, «Particularités lexicales et variétés du français : l'enjeu de l'instrumentalisation lexicographique. À propos des inventaires du Sud et du français en Afrique Noire », dans Michel Francard (dir.), Le régionalisme lexical. De Boeck Supérieur, 1995, pp. 159-170. 
Lopes ». Études littéraires africaines, ${ }^{\circ}$ 45, 2018, pp. 103-113.

Lopes, Henri, Allocution prononcée à l'occasion de l'obtention d'un doctorat honoris causa, le 26 avril, 2002, Université Laval.

Lopes, Henri, Le chercheur d'Afriques, Paris, Éditions du Seuil, 1990.

Manifestes « francophones », Études littéraires africaines, n 48, 2019, pp. 193206.

Maunick, Édouard, «Le territoire d'Henri Lopes », Notre Librairie, Littérature Congolaise, $\mathrm{n}^{\circ}$ 92-93, 1988, pp. 128-131.

Mopoho, Raymond, « Traitement traductologique de l'altérité linguistique dans la production littéraire d'Afrique francophone », présentée au trente et unième Congrès du CIEF en Martinique, juin 2017.

Mukasonga, Scholastique, La femme aux pieds nus, Paris, Gallimard, 2008.

Ngadi Maïssa, Laude, « La mondialité de la langue française dans les

Nzesse, Ladislas, "Oralité et appropriation du français dans la littérature africaine : le cas de Allah n'est pas obligé d'Ahmadou Kourouma et de La joie de vivre de Patrice Nganang », Synergies Algérie, $\mathrm{n}^{\circ}$ 10, 2010, pp. 245-258.

Surmont (de), Jean-Nicolas, «Variationnisme et essentialisme au sein des dictionnaires récents de langue française : quelle vision du français québécois ? » Neuphilologische Mitteilungen, Vol. 109, $\mathrm{n}^{\circ}$ 3, 2008, pp. 361-371. 